\title{
Defect detection of gold-plated surfaces on PCBs using Entropy measures
}

\author{
D. M. Tsai and B. T. Lin \\ Machine Vision Lab. \\ Department of Industrial Engineering and Management \\ Yuan-Ze University, Chung-Li, Taiwan, R.O.C. \\ E-mail: iedmtsai@saturn.yzu.edu.tw
}

\section{Introduction}

Automatic visual inspection algorithms for printed circuit boards (PCBs) have long been focused on geometrical defects of conductive paths [1-4]. Less attention has been paid to the defect inspection of plated surfaces on PCBs. In this paper, we propose two entropy measures of chromatic and directional regularities for the automatic defect inspection of gold-plated fingers (edge connectors) on PCBs.

Connector fingers are metallic pads at the edge of a PCB, which plug into an external socket. The fingers are often plated with gold in order to ensure a good electrical interface for interconnecting external modules, and provide resistance to oxidation [5]. The gold contacts need to be inspected after plating to ensure that an adequate coating of gold has been applied. Figures 1(a)-1(d) show the defect types commonly found on gold-plated surfaces. A layer of gold that is too thin (Figure 1(a)) or contains pinholes (Figure 1(b)) causes the underlying copper to corrode. Too great a current density in the plating process can result in a very rough or "burned" surface as the one shown in Figure 1(c). It can be seen from Figure 1 that the gold-plated surface is not uniform in the image, but involves horizontal texture. The inspection process is far more complicated than the simple binary thresholding of 
a gray level image. Figures 2(a)-2(d) show the binarization results of the gray-level versions of gold finger images in Figures 1(a)-1(d), using the well-known Otsu's thresholding method [6]. The resulting binary images contain horizontally textured patterns along with randomly distributed noise, and make the discrimination between defects and noise difficult.

The gold-plated surfaces of edge fingers can be considered as a homogeneous texture in the image. Traditional texture analysis techniques such as co-occurrence matrix methods $[7,8]$ in the spatial domain, and Fourier-based textural features in the spectral domain $[9,10]$ are too computationally expensive to develop an efficient inspection system for gold-plated surfaces. In this study, we develop two efficient entropy measures to evaluate chromatic and directional anomalies of gold-plated surfaces. Since the pioneer work of Shannon [11], entropy has been used as a measure of complexity of information content in a signal. In image analysis, entropy of gray-level histogram has been used as a measure for texture analysis [12-14]. Desoky and Hall [12], and Jernigan and D' Astous [15] developed entropy techniques that measure the distribution of spectral components of a gray-level image in the frequency domain. They reported that the entropy based on power spectrum is more useful than the one based on gray-level histogram for describing changes in texture. The main disadvantage of the frequency-based entropy method is the computational burden of the Fourier transform.

The traditional entropy measures for texture analysis are solely relied on the information of gray level distribution in an image. They are not appropriate for gold-plated surface inspection because they ignore both chromatic information and structural variation. Therefore, in this study we develop two entropy measures to 
evaluate the regularities of chromatic and directional distributions of gold-plated fingers in a color image. One entropy measure uses two chromatic features extracted from a color model to detect color anomalies such as copper exposure, and the other measure uses edge angles to detect structural defects such as roughness on gold-plated surfaces.

This paper is organized as follows: Section 2 first describes the entropy of chromatic distribution in the CIELUV color space, and then defines the entropy of directional distribution based on Sobel edge gradients. Section 3 presents the experimental results for evaluating the efficacy of the proposed entropy measures. The paper is concluded in Section 4.

\section{Entropy measures of regularity}

Entropy can be used as a measure of regularity of information in a signal. Let $x$ be a discrete random variable whose possible values are $x_{i}, i=1,2, \ldots, n$, and $P\left(x_{i}\right)$ the probability of $x_{i}$. The entropy of the random variable $x$ is defined as

$$
E_{x}=-\sum_{i=1}^{n} P\left(x_{i}\right) \cdot \log P\left(x_{i}\right)
$$

$E_{x}$ quantifies the amount of information of the random variable $x$. Note that $E_{x}$ is always positive or null. $E_{x}$ is null only if among all the possible random values there is one with $P\left(x_{i}\right)=1 . \quad E_{x}$ is maximum when all random values have the same probability, i.e., $P\left(x_{i}\right)=1 / n$. The less uniform the probability distribution, the lower the resultant entropy value, i.e., the lower the entropy value, the higher the 
regularity of an observed signal. In this paper, we propose two entropy measures that quantify individually the average amounts of color and directional distributions in gold-plated surfaces. These two entropy measures are described separately in the following subsections.

\subsection{Entropy of chromatic distribution}

Traditional entropy measure of information in an image is solely based on the gray-level histogram. Chromatic information of textured images is not fully utilized to enhance the discrimination. Many defects found on gold-plated surfaces such as pinhole and copper exposure can be considered as local color variations that break homogeneity in a color image. The entropy of chromatic distribution in a color image can be estimated from its color histogram. In this study, the CIELUV color space [16] is used for color feature representation. The CIELUV space requires an intermediate transform to the $X Y Z$ space from the system-dependent $R G B$ space. The transform for NTSC color vision sensor is given by

$$
\left[\begin{array}{l}
X \\
Y \\
Z
\end{array}\right]=\left[\begin{array}{lll}
0.607 & 0.174 & 0.200 \\
0.299 & 0.587 & 0.114 \\
0.000 & 0.066 & 1.116
\end{array}\right]\left[\begin{array}{l}
R \\
G \\
B
\end{array}\right]
$$

The $C I E L U V$ equation is then applied for tristimulus values $X, Y$ and $Z$ :

$$
\begin{aligned}
& L^{*}=116\left(Y / Y_{n}\right)^{1 / 3}-16 \\
& u^{*}=13 L^{*}\left(u^{\prime}-u_{n}^{\prime}\right) \\
& v^{*}=13 L^{*}\left(v^{\prime}-v_{n}^{\prime}\right)
\end{aligned}
$$


where

$$
\begin{aligned}
& u^{\prime}=\frac{4 X}{X+15 Y+3 Z} \\
& v^{\prime}=\frac{9 Y}{X+15 Y+3 Z}
\end{aligned}
$$

$Y_{n}, u_{n}^{\prime}$, and $v_{n}^{\prime}$ are the chromaticity coordinates of the reference white. $L^{*}$ represents lightness. The $u^{*}$ and $v^{*}$ dimensions correlate with red-green and yellow-blue chroma perceptions.

The well-known and commonly used color space CIELAB was also originally adopted for color transformation in this study. An empirical study showed that CIELUV and CIELAB perform similarly in many cases for gold-plated surface inspection, but CIELUV is slightly better than CIELAB in terms of the detected defect sizes. The CIELUV color space can also be represented in terms of cylindrical coordinates, which provide predictors of chroma $C_{u v}^{*}$ and hue $h_{u v}$ as expressed below:

$$
\begin{aligned}
& C_{u v}^{*}=\left[\left(u^{*}\right)^{2}+\left(v^{*}\right)^{2}\right]^{1 / 2} \\
& h_{u v}=\tan ^{-1}\left(\frac{v^{*}}{u^{*}}\right)
\end{aligned}
$$

The entropy measure of chromatic distribution in an image can be calculated from the color histogram in 1-D (single color feature), 2-D (two color features) and 3-D (all three color features of a color space). The resolution of the color histogram can be chosen by specifying the number of bins along each axis of color features. In 1-D color histogram, the hue $h_{u v}$ is used to evaluate the chromatic regularity of the gold-plated surface. Let the axis of color feature $h_{u v}$ be divided into $N_{c}$ subintervals of equal width. Denote $f_{c}(i)$ by the number of pixels falling in the 
subinterval $i, i=1,2, \ldots, N_{c}$. The probability of a color in subinterval $i$ can be calculated by

$$
P_{c}(i)=\frac{f_{c}(i)}{R \cdot C}
$$

where $R \times C$ is the effective image size used for evaluating the regularity. The color entropy with a single color feature is defined by

$$
E_{c 1}=-\sum_{i=1}^{N_{c}} P_{c}(i) \cdot \log P_{c}(i)
$$

In 2-D color histogram, color features $u^{*}$ and $v^{*}$ are adopted for evaluating color regularity. The $u^{*}$ and $v^{*}$ axes of the $\left(u^{*}, v^{*}\right)$ space are individually partitioned into $N_{c}$ subintervals of equal width. Given an image of size $R \times C$, let $f_{c}(i, j)$ be the number of pixels that fall within the $(i, j)^{\text {th }}$ grid in the $\left(u^{*}, v^{*}\right)$ space, $i, j=1,2, \ldots, N_{c}$. The probability of a color falling in the $(i, j)^{\text {th }}$ grid is given by

$$
P_{c}(i, j)=\frac{f_{c}(i, j)}{R \cdot C}
$$

The color entropy with two color features $\left(u^{*}, v^{*}\right)$ is defined by

$$
E_{c 2}=-\sum_{i=1}^{N_{c}} \sum_{j=1}^{N_{c}} P_{c}(i, j) \cdot \log P_{c}(i, j)
$$

The 3-D color histogram can be constructed in a similar way as the 2-D one by including all three color features $L^{*}, u^{*}$ and $v^{*}$ of the CIELUV space. Our empirical study showed that $E_{c 2}$ (color entropy with two color features) and $E_{c 3}$ (color entropy with three color features) measures perform equally well for gold-plated surface inspection. However, the computation time of $E_{c 3}$ is more than 70 times over that of $E_{c 2}$. 
In order to evaluate the effectiveness of entropy measures $E_{c 1}$ and $E_{c 2}$, Figure 3(a1)-3(a5) show, respectively, the images of a single color band, three discrete color bands, five discrete color bands, ten discrete color bands, and a plate with continuous color spectrum ranging from violet to red. The entropy values of $E_{c 1}$ and $E_{c 2}$ as a function of the number of color bands in an image are depicted in Figure 3(b). The corresponding entropy statistics are summarized in Table 1. It can be seen from Figure 3(b) that the entropy value gets larger as the number of colors added to the image increases for both $E_{c 1}$ and $E_{c 2}$. The plot of $E_{c 2}$ is quite steep, whereas the plot of $E_{c 1}$ is relatively flat. The entropy measure with two color features $\left(E_{c 2}\right)$ has better discrimination to detect chromatic variations, compared to the entropy measure with single color feature $\left(E_{c 1}\right)$. Figure 4(a) shows a gold finger (marked with a rectangular frame) with a void defect, and Figure 4(b) presents a plot of the $E_{c 2}$ function in 3-D perspective. It shows that the void is notably high in the 3-D plot. Based on the consideration of detection effectiveness and computational efficiency, entropy measure $E_{c 2}$ is the best choice for detecting color variation in gold-plated surfaces.

\subsection{Entropy of directional distribution}

Nick defects on gold-plated surfaces cause not only chromatic variation in color but also significant directional variation in structure. A rough (or "burned") surface due to excessive current density in the plating process may not show significant color variation, but may present structure variation in an image. The measure of directional regularity using entropy proceeds as follows.

Let $R(x, y), G(x, y)$ and $B(x, y)$ denote the $R, G$ and $B$ stimulus values at 
pixel coordinates $(x, y)$, respectively. The intensity at pixel coordinates $(x, y)$ is defined by

$$
f(x, y)=\frac{1}{3}[R(x, y)+G(x, y)+B(x, y)]
$$

The directional angle at pixel $(x, y)$ is given by the Sobel gradient operator [17]:

$$
\theta(x, y)=\tan ^{-1}\left[\frac{G_{Y}(x, y)}{G_{X}(x, y)}\right]
$$

where

$$
\begin{aligned}
G_{X}(x, y)= & {[f(x-1, y+1)+2 f(x, y+1)+f(x+1, y+1)]-} \\
& {[f(x-1, y-1)+2 f(x, y-1)+f(x+1, y-1)] } \\
G_{Y}(x, y)= & {[f(x+1, y-1)+2 f(x+1, y)+f(x+1, y+1)]-} \\
& {[f(x-1, y-1)+2 f(x-1, y)+f(x-1, y+1)] }
\end{aligned}
$$

Divide the full range of directional angle $\theta$ into $N_{\theta}$ subintervals of equal width. Denote $f_{\theta}(i)$ by the number of edge points falling in the subinterval $i$, $i=1,2, \ldots, N_{\theta}$. The probability of directional angle in the subinterval $i$ can be calculated by

$$
P_{\theta}(i)=\frac{f_{\theta}(i)}{\sum_{j=1}^{N_{\theta}} f_{\theta}(j)}
$$

The entropy measure of directional distribution is given by

$$
E_{\theta}=-\sum_{i=1}^{N_{\theta}} P_{\theta}(i) \cdot \log P_{\theta}(i)
$$

In order to evaluate the effectiveness of the direction entropy $E_{\theta}$, Figures 5(a1)-5(a4) show the line-patterned images that contain one, two, three and four directions, respectively. Figure 5(a5) is an isotropic texture of sandpaper. The plot 
of $E_{\theta}$ values as a function of the number of directions is presented in Figure 5(b). It shows that the $E_{\theta}$ value increases consistently as the textural structure becomes complicated. An isotropic texture has the largest $E_{\theta}$ value of 3.58 , while a simple line-patterned texture with a single direction has a small $E_{\theta}$ value of 1.05. Figure 6(a) shows a gold finger (marked with a rectangular frame) with a nick defect. Figure 6(b) presents a plot of the $E_{\theta}$ function in 3-D perspective. It shows that the defective region is visibly higher than the homogeneous region in the 3-D plot. $E_{c 2}$ along with $E_{\theta}$ can be sufficiently used to detect various defects on gold-plated surfaces.

\section{Experimental results}

In this section, we present the experimental results for evaluating the efficacy of the proposed entropy measures for gold-plated surface inspection. In our implementations, all algorithms are programmed in the $\mathrm{C}$ language and executed on a personal computer with a Pentium III-800 Mhz processor. The image size is $640 \times 480$ pixels with eight bits of intensity per color band. In order to obtain a sufficient resolution for inspecting various plating defects, and yet contain as many gold fingers as possible in a sensor image, an input image contains 7 gold fingers, each of $120 \times 45$ pixels. The number of partitioned subintervals of each color feature of $u^{*}$ and $v^{*}$ used in color entropy $E_{c 2}$ is 50 . Therefore, the 2-D color histogram is a $50 \times 50$ matrix. The $\theta$-axis of direction entropy $E_{\theta}$ is divided into subintervals of equal width $5^{\circ}$.

In the experiments, the entropy is computed by sliding a neighborhood window in a pixel-by-pixel basis throughout the entire gold finger regions in the sensor image. 
A pixel defined in a small neighborhood window in a homogeneous gold-plated region will have small entropy value, and the one in any irregular region will yield a large entropy value. Therefore, we employ the simple statistical process control principle to set up the threshold for distinguishing defective regions from homogeneous regions in the resulting entropy image. The threshold used in the experiment is given by

$$
\mu_{E}+k \cdot \sigma_{E}
$$

where $\mu_{E}$ and $\sigma_{E}$ are the mean and standard deviation of entropy values in defect-free sample images. $\quad k$ is a control constant. A 3-sigma standard has been used for detecting anomalies. Pixels with entropy values larger than the threshold $\mu_{E}+3 \sigma_{E}$ will be shown in white, and the ones with entropy values below the threshold will appear as black in the resulting binary image.

In order to evaluate the effect of size changes of neighborhood window, the detection results of $E_{c 2}$ from the window sizes of $7 \times 7,11 \times 11$ and $19 \times 19$ are shown in Figure 7 (pinhole defect) and Figure 8 (nick defect). It can be seen from Figures 7 and 8 that too small the window size can not sufficiently identify all defective pixels, and reduces the size of the detected defect. However, too large the window size may not generate better detection result, and is more computationally expensive. With the image resolution used in the experiment, the neighborhood window size of $11 \times 11$ pixels is effective and efficient to detect the detail of defects in gold-plated surfaces.

In chromatic defect detection, Figures 9(a1)-9(d1) show four test images of gold-plated surfaces that contain various chromatic defects. Without loss visibility, 
all test samples of colored gold fingers are displayed with gray-level images. The detection results from color entropy $E_{c 2}$ are illustrated in Figures $9(\mathrm{a} 2)-9(\mathrm{~d} 2)$. They reveal that the $E_{c 2}$ measure is very effective to identify local anomalies in gold-plated surfaces. In structural defect detection, Figures 10(a1) and 10(b1) show two test images that contain structural defects. It can be seen from Figure 10 that both entropy measures $E_{c 2}$ and $E_{\theta}$ can detect the linear defects, but measure $E_{\theta}$ is slightly better than measure $E_{c 2}$ in terms of the detected shapes of defects.

As aforementioned in the previous section, a rough (or "burned") gold-plated surface due to excessive current density may result in homogeneous color distribution in the whole image, and the color entropy $E_{c 2}$ fails to identify the rough surface. In roughness detection, Figures 11(a)-11(c) show respectively the images of a defect-free surface, a fine surface with a local defect of copper exposure, and a burned surface. The mean and standard deviation of $E_{\theta}$ values for each gold finger from left to right in each of the three images are tabulated in Table 2. It shows that the mean $E_{\theta}$ value for fine surfaces as the ones shown in Figures 11(a) and 11(b) is between 0.1 and 0.5 , whereas the mean $E_{\theta}$ value for the burned surface is distinctly larger than 1.0. Figure 11(d) displays the processing result of the burned surface, in which the pixels with $E_{\theta}$ value larger than 0.7 are shown in white and the remaining pixels appear as black. The entropy measure $E_{\theta}$ gives distinct discrimination between regular and rough surfaces. 


\section{Conclusions}

Many automated visual inspection techniques for printed circuit boards have been developed in the past years. However, most of the inspection methods use only gray-level information of PCB images and focus mainly on geometrical defects of conductive paths. In this paper, we have proposed two entropy measures for surface inspection of gold fingers on PCBs. The entropy measure $E_{c 2}$ uses two color features $u^{*}$ and $v^{*}$ from the CIELUV color space to detect chromatic anomalies, and the entropy measure $E_{\theta}$ uses edge angles to detect structural variations. Experimental results reveal that entropy measure $E_{c 2}$ alone is sufficient to detect various chromatic and structural defects on gold-plated surfaces, except for the roughness defect. The entropy measure $E_{\theta}$ is ideally suited for identifying the rough plating surface since a regular surface and a coarse one yield distinct values of $E_{\theta}$.

With the given system setup in the experiment (a gold finger of size $120 \times 45$ pixels and 7 edge fingers in one image; neighborhood window of size $11 \times 11 ; 50$ partitioned subintervals for axes $u^{*}$ and $v^{*}$, and 36 subintervals for axis $\theta$ ), the computation time is within 1 second. It is competitive with the inspection time of human personnel. On-line inspection of gold-plated fingers can be realized with the two entropy measures $E_{c 2}$ and $E_{\theta}$. 


\section{References}

1. Y. Hara, N. Akiyama and K. Karasaki, "Automatic inspection system for printed circuit boards," IEEE Transactions on Pattern Analysis and Machine Intelligence, 5, pp. 623-630, 1983.

2. M. Mogant and F. Ercal, "Automatic PCB inspection algorithms: a survey," Computer Vision and Image Understanding, 63, pp. 287-313, 1996.

3. M. Mogant and F. Ereal, "A subpattern level inspection system for printed circuit board," Computer Vision and Image Understanding, 70, pp. 51-62, 1998.

4. C. -S. Lin and L. W. Lue, "Image system for fast positioning and accuracy inspection of ball grid array boards," Microelectronics and Reliability, 41, pp. 119-128, 2001.

5. J. Beamish, "Printed circuit board fabrication procedures," http://www.mansys.herts.ac.uk/john/pcbstuff/PCBFabrication.html, University of Hertfordshire, UK.

6. U. M. Otsu, "A thresholding selection method from gray-level histogram," IEEE Transactions on System, Man and Cybernetics, SMC-3, pp. 62-66, 1979.

7. L. H. Siew, R. M. Hodgson and E. J. Wood, "Texture measures for carpet wear assessment," IEEE Transactions on Pattern Analysis and Machine Intelligence, 10, pp. 92-105, 1988.

8. K. V. Ramana and B. Ramamoorthy, "Statistical methods to compare the texture features of machined surfaces," Pattern Recognition, 29, pp. 1447-1459, 1996.

9. S. S. Liu and M. E. Jernigan, "Texture analysis and discrimination in additive noise," Computer Vision, Graphics and Image Processing, 49, pp. 52-67, 1990.

10. R. Muzzolini, Y. -H. Yang and R. Pierson, "Texture characterization using robust statistics," Pattern Recognition, 27, pp. 119-134, 1994.

11. C. E. Shannon and W. Weaver, The Mathematical Theory of Communication, University of Illinois Press, Urbana, IL, 1949.

12. A. H. Desoky and S. A. Hall, "Entropy measures for texture analysis based on Hadamard transform," Proceedings of the IEEE SOUTHEASTCON Conference, 2, pp. 467-470, 1990. 
13. C. A. Chang, H.-D. Lin and C.-C. Lo, "Test of pattern change for automated detection of printing faults using computer vision systems," International Journal of Industrial Engineering, 4, pp. 5-13, 1997.

14. B. R. P. Dawson and A. J. Parsons, "Texture measures for the identification and monitoring of urban derelict land," International Journal of Remote Sensing, 15, pp. 1259-1271, 1994.

15. M. E. Jernigan and F. D' Astous, "Entropy-based texture analysis in the spatial frequency domain," IEEE Transactions on Pattern Analysis and Machine Intelligence, 6, pp. 237-243, 1984.

16. M. D. Fairchild, Color Appearance Models, Addison-Wesley, Reading, Mass., 1998.

17. R. C. Gonzalez and R. E. Woods, Digital Image Processing, Addison-Wesley, Reading, Mass., 1992. 


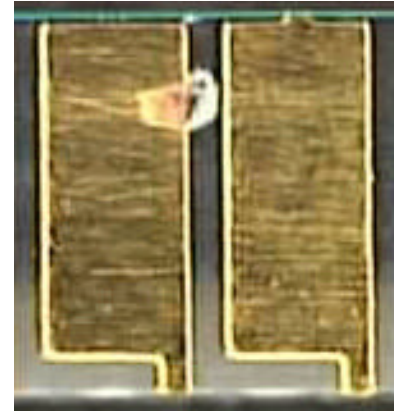

(a)

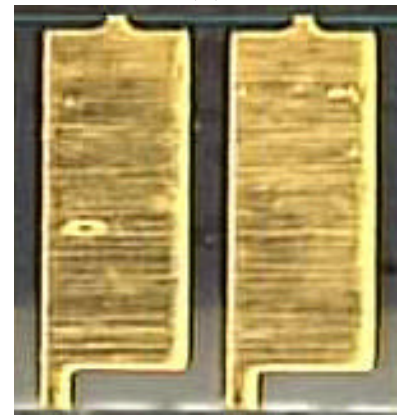

(c)

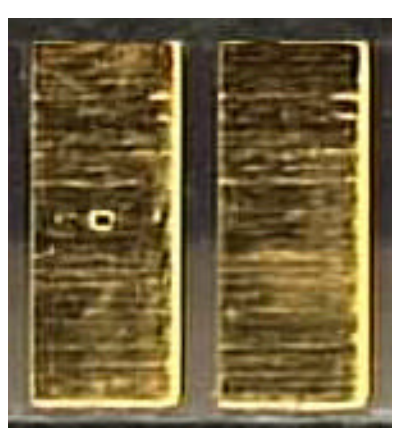

(b)

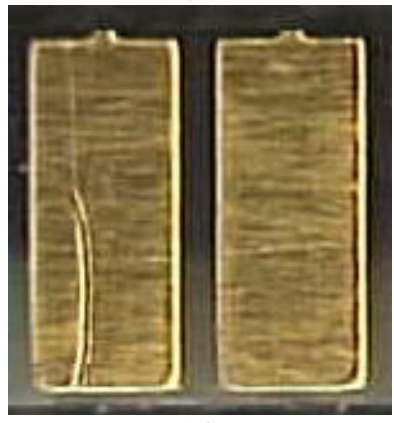

(d)

Figure 1. Common defect types found on gold-plated fingers: (a) Copper exposure. (b) Pinhole. (c) Rough surface. (d) Nick. 


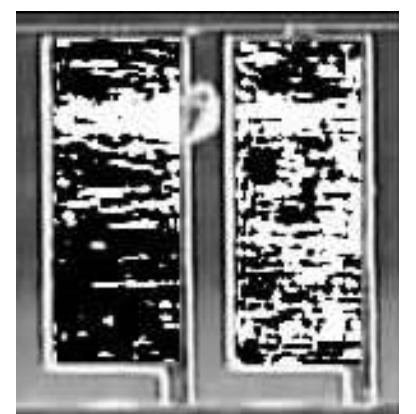

(a)

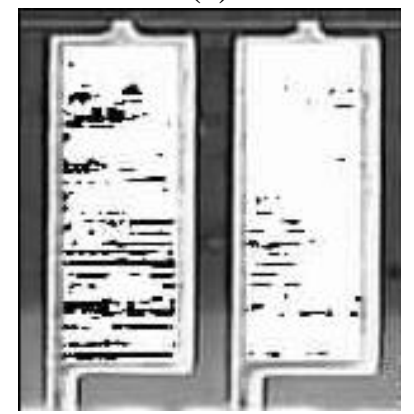

(c)

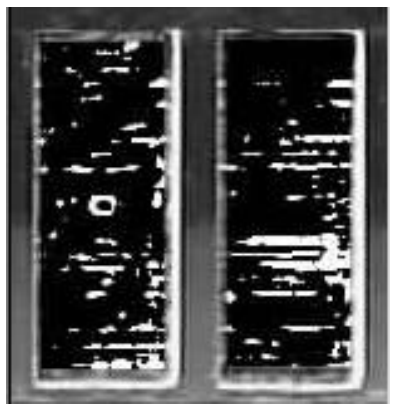

(b)

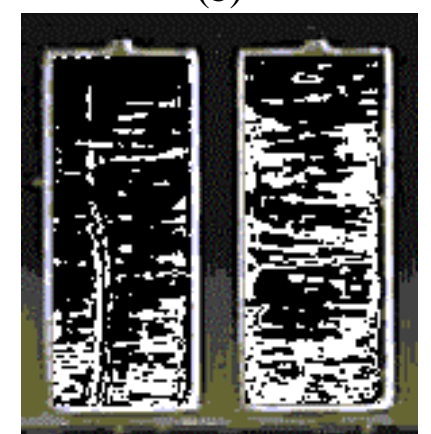

(d)

Figure 2. The resultant binary images corresponding to (a)-(d) in Figure 1, respectively. 


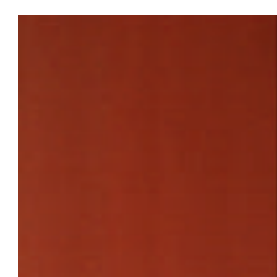

(a1)

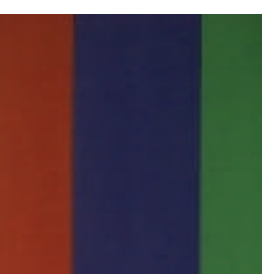

(a2)

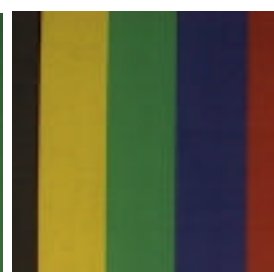

(a3)

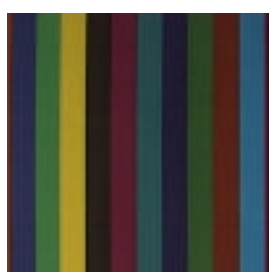

(a4)

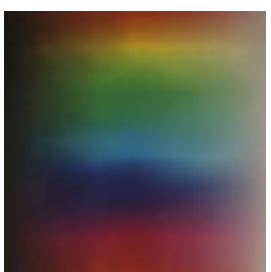

(a5)

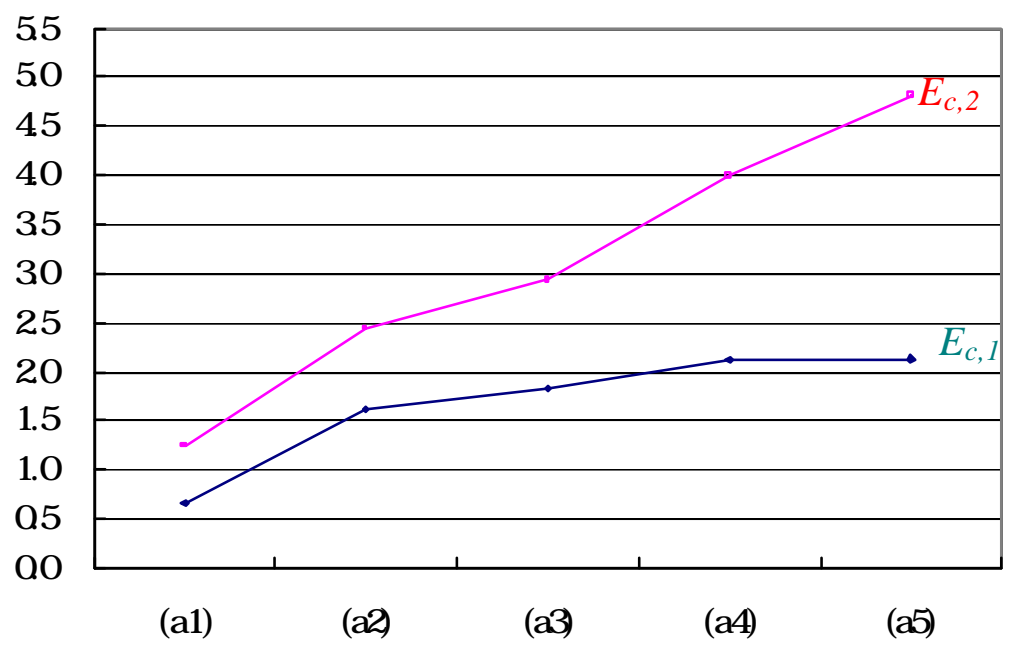

(b)

Figure 3. (a1)-(a5) Color image plates containing one color band, 3 color bands, 5 color bands, 10 color bands and continuous color spectrum, respectively. (b) The plots of $E_{c 1}$ and $E_{c 2}$ as a function of the number of color bands in an image. 


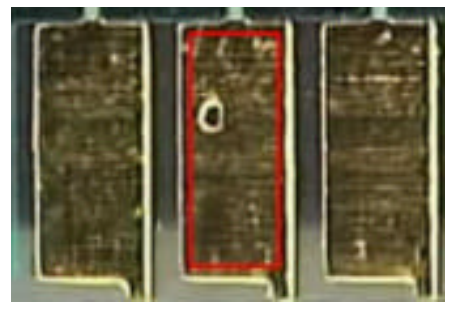

(a)

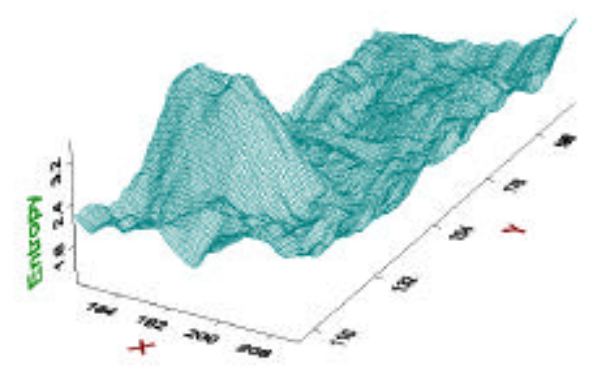

(b)

Figure 4. (a) The image of a gold finger containing a void defect. (b) The corresponding $E_{c 2}$ function in 3-D perspective (the finger in the middle of the image). 


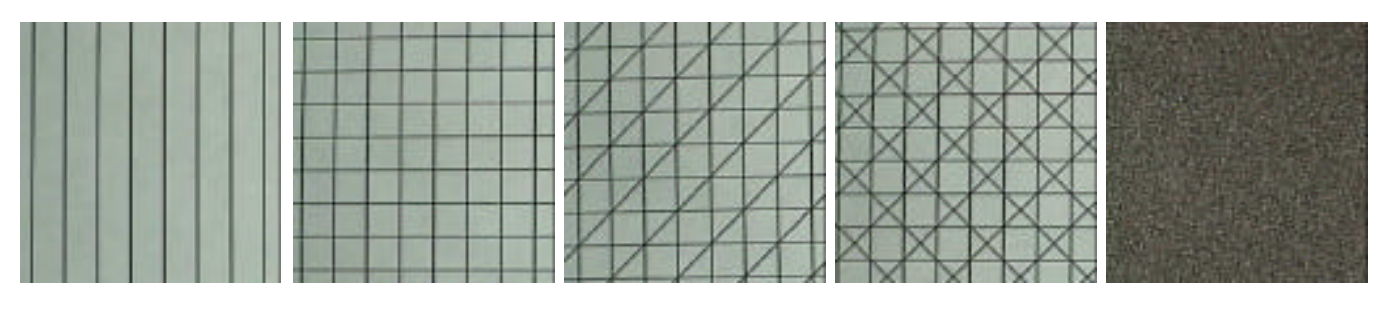
(a1) $E_{\theta}=1.05$
(a2) $E_{\theta}=2.30$
(a3) $E_{\theta}=2.84$
(a4) $E_{\theta}=3.17$
(a5) $E_{\theta}=3.58$

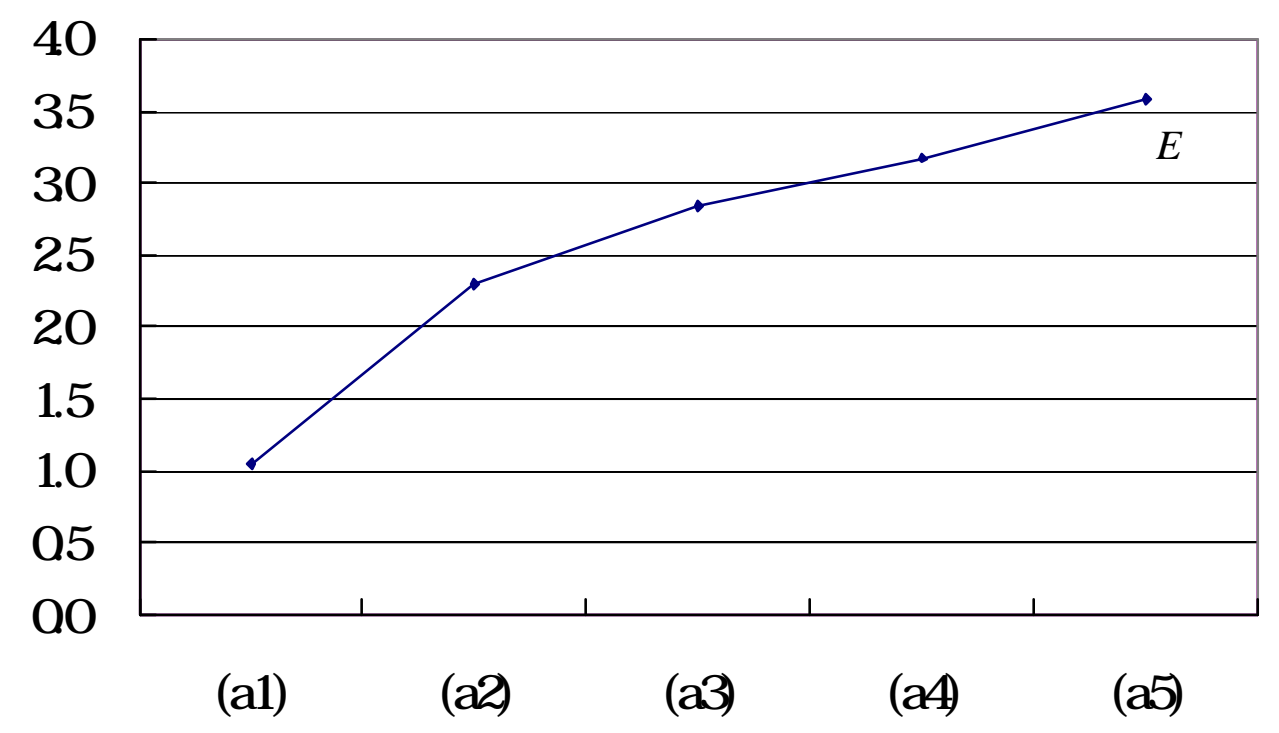

(b)

Figure 5. (a1)-(a4) Line-patterned images containing one, two, three and four directions, respectively. (a5) An isotropic texture of sandpaper. (b) The plot of $E_{\theta}$ as a function of the number of directions in an image. 


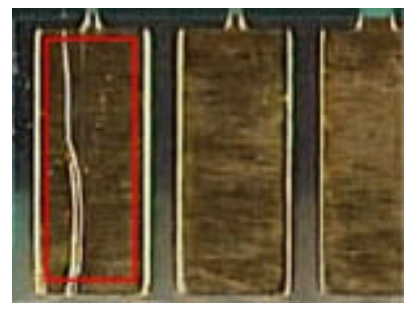

(a)

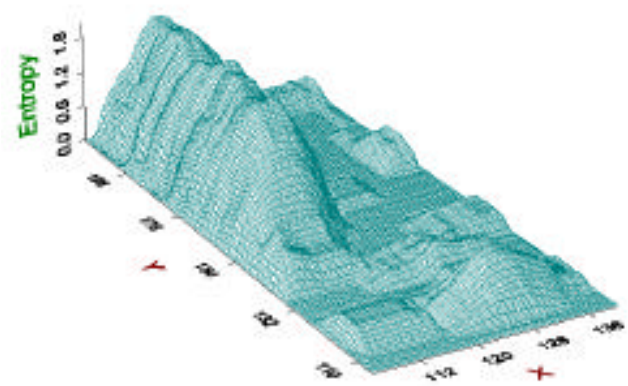

(b)

Figure 6. (a) The image of a gold finger containing a nick. (b) The corresponding $E_{\theta}$ function in 3-D perspective (the left finger in the image). 


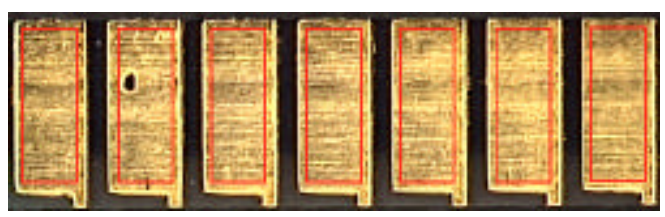

(a)

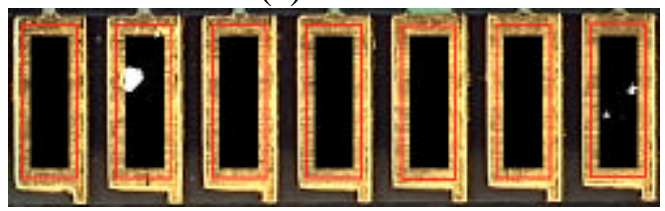

(c) $11 \times 11$

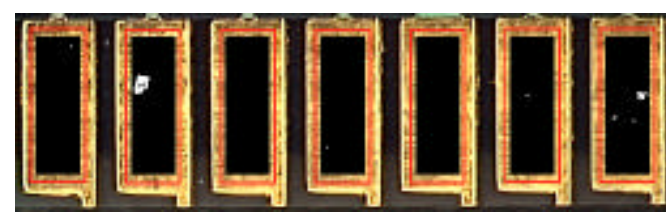

(b) $7 \times 7$

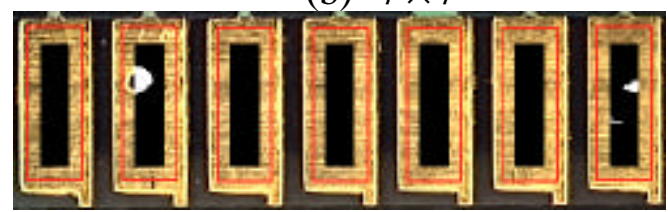

(d) $19 \times 19$

Figure 7. Effect of neighborhood window sizes for the gold fingers with a pinhole: (a) The original image. (b)-(d) The processing results from window sizes $7 \times 7$, $11 \times 11$ and $19 \times 19$, respectively.

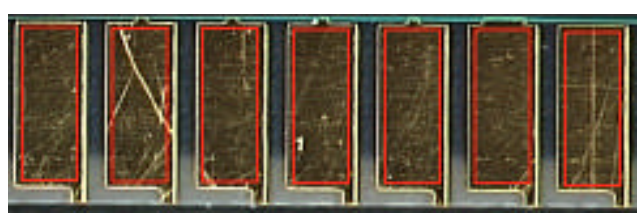

(a)

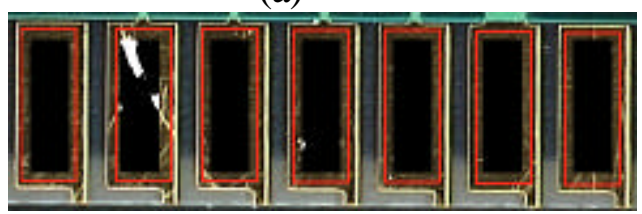

(c) $11 \times 11$

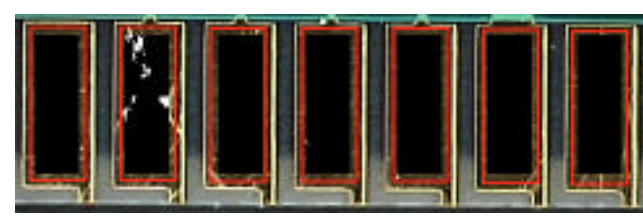

(b) $7 \times 7$

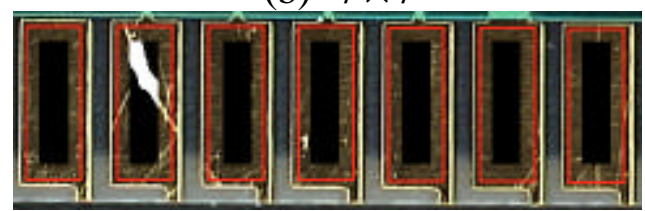

(d) $19 \times 19$

Figure 8. Effect of neighborhood window sizes for the gold fingers with a nick: (a) The original image. (b)-(d) The processing results from window sizes $7 \times 7$, $11 \times 11$ and $19 \times 19$, respectively. 


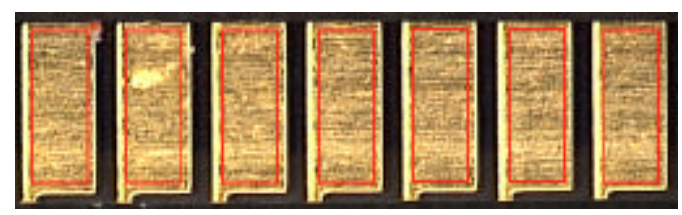

(a1)

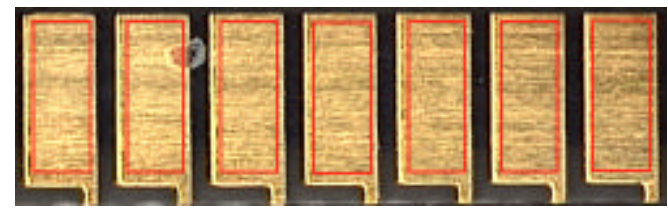

(b1)

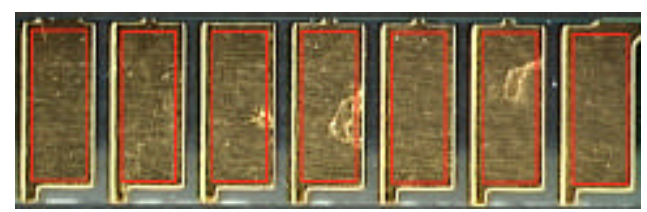

(c1)

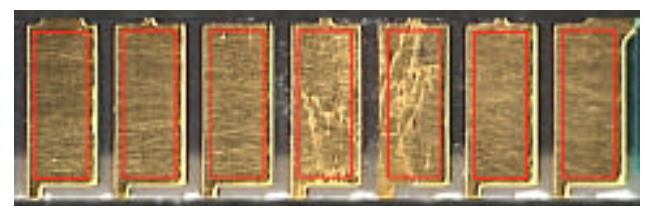

(d1)

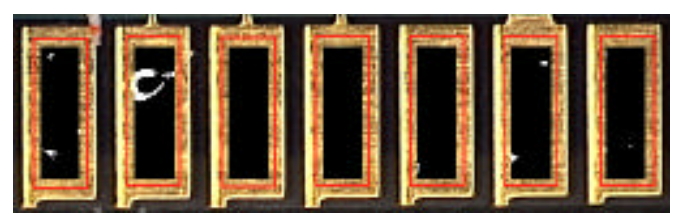

(a2)

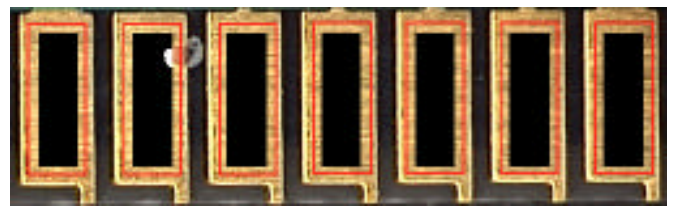

(b2)

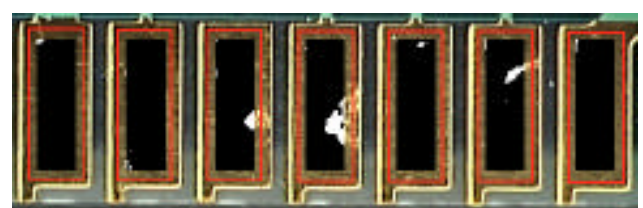

(c2)

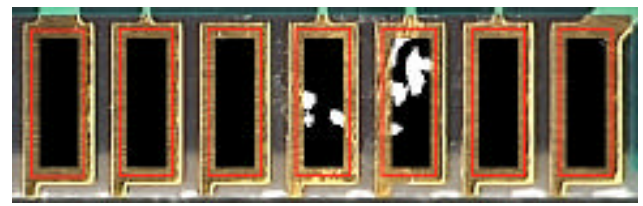

$(\mathrm{d} 2)$

Figure 9. (a1)-(d1) The gold finger images with various chromatic defects on the surfaces. (a2)-(d2) The detection results of the corresponding images (a1)-(d1), respectively. 


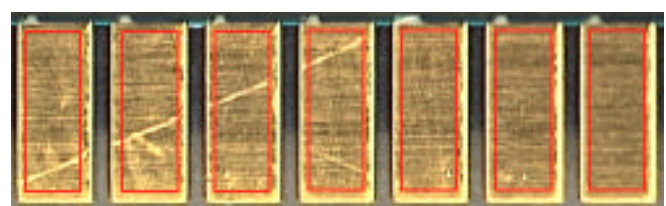

(a1)

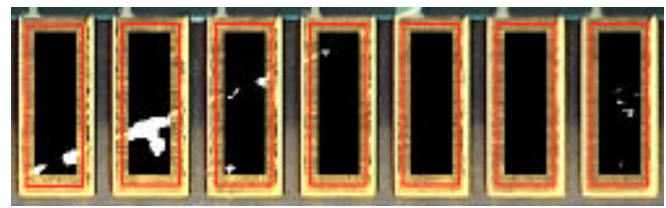

(a2) $E_{c 2}$

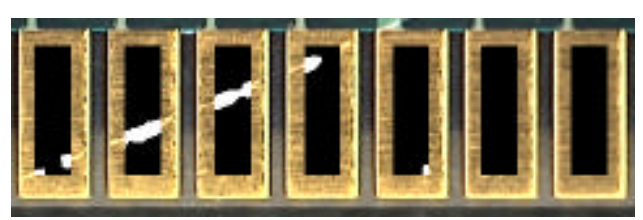

(a3) $E_{\theta}$

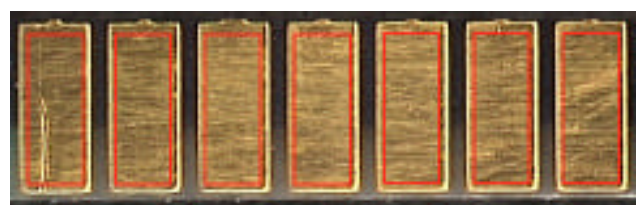

(b1)

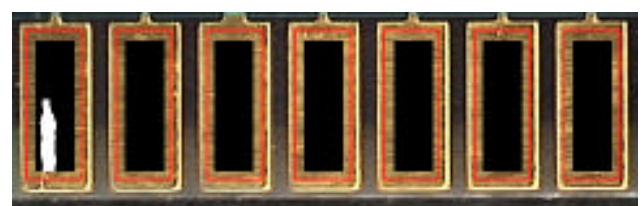

(b2) $E_{c 2}$

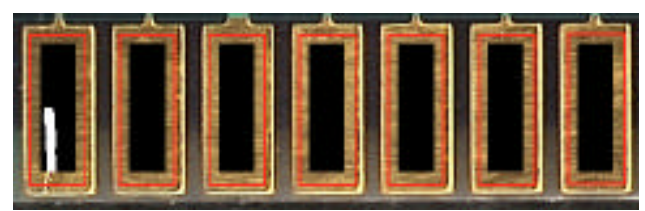

(b3) $E_{\theta}$

Figure 10. (a1), (b1) The gold fingers with structural defects. (a2), (b2) The corresponding detection results from entropy $E_{c 2}$. (a3), (b3) The corresponding detection results from entropy $E_{\theta}$. 


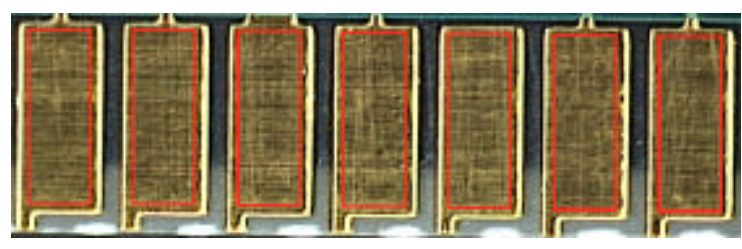

(a)

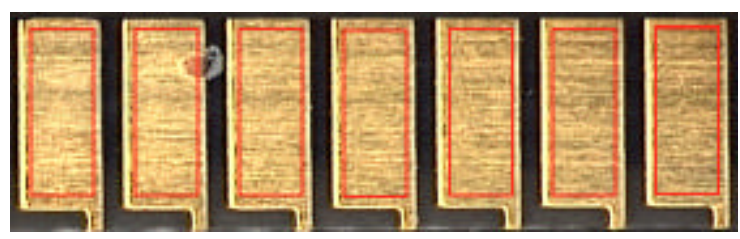

(b)

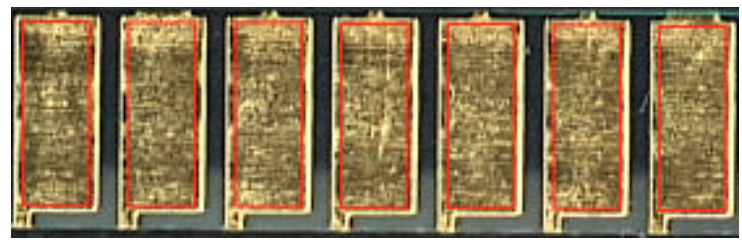

(c)

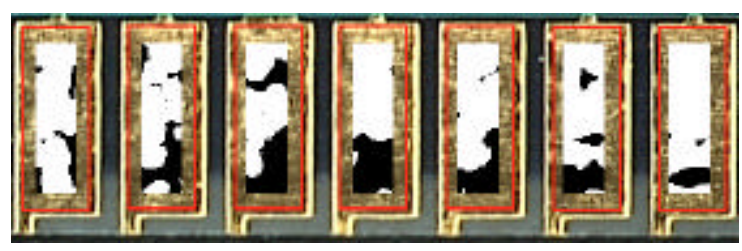

(d)

Figure 11. The sample images used for evaluating the $E_{\theta}$ values of rough surfaces: (a) Fine gold-plated surfaces without defects. (b) Fine surfaces with exposed copper. (c) Rough(burned) surface. (d) The binarization result of (c). 
Table 1. The entropy statistics for color images in Figures 3(a1)-(a5).

\begin{tabular}{|c|c|c|c|c|c|}
\hline \multirow{2}{*}{$\begin{array}{l}\text { Entropy } \\
\text { measure }\end{array}$} & \multicolumn{5}{|c|}{ Color plates } \\
\hline & Fig. 3(a1) & Fig. 3(a2) & Fig. 3(a3) & Fig. 3(a4) & Fig. 3(a5) \\
\hline$E_{c 1}$ & 0.67 & 1.61 & 1.83 & 2.12 & 2.13 \\
\hline$E_{c 2}$ & 1.25 & 2.43 & 2.93 & 3.99 & 4.81 \\
\hline
\end{tabular}

Table 2. The $E_{\theta}$ statistics of images with varying surface roughness.

\begin{tabular}{|l|l|l|l|l|l|l|l|l|}
\hline \multirow{2}{*}{ Image } & \multirow{2}{*}{ Statistics } & \multicolumn{7}{|l|}{ Gold finger number (from left to right) } \\
\cline { 3 - 10 } & & 1 & 2 & 3 & 4 & 5 & 6 & 7 \\
\hline \multirow{2}{*}{ Fig. 11(a) } & Mean & 0.07 & 0.28 & 0.53 & 0.53 & 0.26 & 0.15 & 0.16 \\
\cline { 2 - 10 } & Std.dev. & 0.09 & 0.28 & 0.46 & 0.40 & 0.29 & 0.16 & 0.19 \\
\hline \multirow{2}{*}{ Fig. 11(b) } & Mean & 0.11 & 0.16 & 0.11 & 0.08 & 0.26 & 0.32 & 0.32 \\
\cline { 2 - 10 } & Std.dev. & 0.27 & 0.36 & 0.21 & 0.20 & 0.37 & 0.40 & 0.33 \\
\hline \multirow{2}{*}{ Fig. 11(c) } & Mean & 1.51 & 1.54 & 1.68 & 1.66 & 1.58 & 1.52 & 1.39 \\
\cline { 2 - 9 } & Std.dev. & 0.62 & 0.58 & 0.65 & 0.66 & 0.50 & 0.54 & 0.62 \\
\hline
\end{tabular}

